\title{
Flight of the PEGASUS? Comparing Transformers on Few-Shot and Zero-Shot Multi-document Abstractive Summarization
}

\author{
Travis R. Goodwin and Max E. Savery and Dina Demner-Fushman \\ U.S. National Library of Medicine \\ National Institutes of Health \\ \{firstname. lastname\}@nih.gov
}

\begin{abstract}
Recent work has shown that pre-trained Transformers obtain remarkable performance on many natural language processing tasks, including automatic summarization. However, most work has focused on (relatively) data-rich single-document summarization settings. In this paper, we explore highly-abstractive multi-document summarization, where the summary is explicitly conditioned on a user-given topic statement or question. We compare the summarization quality produced by three state-of-the-art transformer-based models: BART, T5, and PEGASUS. We report the performance on four challenging summarization datasets: three from the general domain and one from consumer health in both zero-shot and few-shot learning settings. While prior work has shown significant differences in performance for these models on standard summarization tasks, our results indicate that with as few as 10 labeled examples, there is no statistically significant difference in summary quality, suggesting the need for more abstractive benchmark collections when determining state-of-the-art.
\end{abstract}

\section{Introduction}

Since its inception (Luhn, 1958), automatic summarization has focused on summarizing documents either in a generic way - conveying the main points of the document to any reader regardless of their information need - or in a task-specific way - distilling the important points of the document with respect to a specific information need such as a question or topic statement (Mani, 2009). In the latter case, the selection of the most salient points in the document (i.e., content selection) as well as the expression of those points (i.e., surface realization) must be explicitly conditioned on a user-given natural language context statement, such as a question or topic of interest. In this setting, a single passage may be summarized in different ways depending on the context description. Consequently, obtaining reference summaries is often time- or cost-prohibitive, particularly when dealing with specialized domains such as healthcare.

The Document Understanding Conference has explored Topic-driven summarization (DUC) and its successor, the Text Analysis Conference (TAC), which both ran community evaluations of topic- or question-based summarization. Specifically, participants were asked to develop automatic summarization approaches for generating single- or multi-document summaries that summarized a set of documents with respect to a given topic description or question, as shown in Figure 1. Human assessors manually judged submitted summaries.

In this work, we revisit the multi-document topic-driven abstractive summarization datasets produced from DUC 2007, TAC 2009, and TAC 2010, as well as question-driven summarization from consumer health. Because these datasets are relatively small (approximately 45 topics each), we explore modern transformer-based models' performance in the zero-shot and few-shot (10 examples) learning settings. Specifically, we explore the quality of multi-document abstractive summarization generated by T5 (Raffel et al., 2019), BART (Lewis et al., 2019), and PEGASUS (Zhang et al., 2019).

This work is licensed under a Creative Commons Attribution 4.0 International License. License details: http: //creativecommons.org/licenses/by/4.0/. 


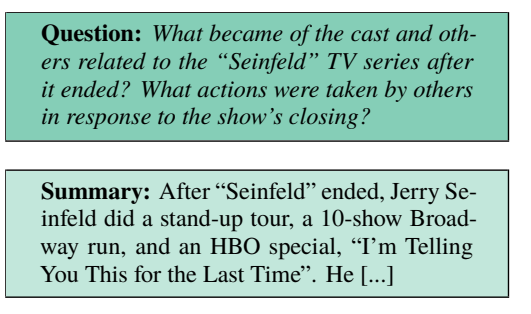

(a) DUC 2007

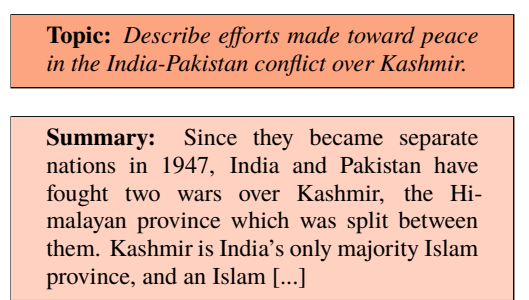

(b) TAC 2009

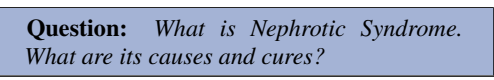

What are its causes and cures?

Summary: Nephrotic syndrome is caused by different disorders that damage the kidneys. In adults, most commonly by glomerulonephritis. This damage leads to the release of too much protein in the urine [...]

(c) MEDIQA

Figure 1: Example topic- and question-driven multi-document abstractive summaries (documents omitted).

\section{Background}

Recent work has indicated that transfer learning (pre-training a model on data-rich tasks before fine-tuning it on a downstream task) obtains remarkable performance on many natural language processing tasks (Yang et al., 2019; Dong et al., 2019; Liu et al., 2019b). The most successful models are obtained through self-supervised pre-training with massive datasets to obtain transferable knowledge for new tasks (i.e., fine-tuning) with less abundant data (Devlin et al., 2019; Lewis et al., 2019; Keskar et al., 2019; Raffel et al., 2019). More recently, research has indicated that these models can generate language conditioned on a user-given prompt or context. For example, this prompt can guide the model's content selection towards a particular topic (Keskar et al., 2019) or inform surface realization for a specific task (Lewis et al., 2019; Raffel et al., 2019). In Liu et al. (2020), the authors condition an extractive transformer using "control codes" to specify the position, importance, and diversity of the sentences in the source text. In this work, we adapt this paradigm to train and evaluate BART, T5, and PEGASUS for abstractive multi-document summarization.

Although zero-shot learning (ZSL) has received considerable attention in the image processing community, there has been comparatively little work on zero-shot learning specifically for summarization: Duan et al. (2019) explore zero-shot learning for cross-lingual sentence summarization and Liu et al. (2019a) explored zero-shot abstractive summaries of five-sentence stories. We extend these works by evaluating zero-shot and few-shot learning for multi-document abstractive summarization.

\section{Models}

In this work, we compare three of the most prominent conditional language generation models: T5, BART, and PEGASUS. To facilitate comparison, for each model we chose the variant with the most similar architecture (such that each consists of 12 transformer layers and a similar number of learnable parameters). Each model is pre-trained with unique strategies as described below.

BART (Bidirectional and Auto-Regressive Transformers) is pre-trained on document rotation, sentence permutation, text-infilling, and token masking and deletion objectives (Lewis et al., 2019). In our experiments, we used BART-Large.

T5 (Text-to-Text Transfer Transformer) is pre-trained on several unsupervised and supervised objectives, such as token and span masking, as well as translation, classification, reading comprehension, and summarization. Importantly, each objective is treated as a language-generation task, where the model is conditioned to generate the correct output based on a textual prompt included in the input sequence (Raffel et al., 2019). In this work, we used T5-Base.

PEGASUS (Pre-training with Extracted Gap-sentences for Abstractive SUmmarization Sequence-tosequence) was specifically designed for abstractive summarization and is pre-trained with a self-supervised gap-sentence-generation objective (Zhang et al., 2019). In this task, entire sentences are masked from the source document, concatenated, and used as the target "summary". We used PEGASUS-Base in our experiments. 


\section{Experiments}

We evaluated T5, BART, and PEGASUS in zero-shot (ZSL) and few-shot (FSL) learning settings on four datasets. Summary quality was measured using ROUGE-1, ROUGE-2, and ROUGE-L $F_{1}$-scores (Lin, 2004); BLEU-4 (Papineni et al., 2002); and Repetition Rate (in unigrams). Implementation details are provided in Appendix A.

\subsection{Answer Summarization at DUC 2007}

The 2007 challenge of the Document Understanding Conference (DUC) focused on answering 45 natural language questions by summarizing sets of 10 documents from the AQUAINT English news corpus (Graff, 2002). Reference summaries were between 230 and 250 words. We used 30 topics for testing (with 10 for training and 5 for validation under FSL). Table 1 presents these results, showing that BART obtains the highest quality summaries in both settings, though FSL provides a significant increase for all models.

\begin{tabular}{|c|c|c|c|c|c|}
\hline System & ROUGE-1 & ROUGE-2 & ROUGE-L & BLEU-4 & Repetition \\
\hline T5 (ZSL) & $21.21(20.37-22.04)$ & $4.35(3.82-4.91)$ & $11.59(11.17-12.03)$ & $1.45(1.24-1.73)$ & $33.21(31.82-34.60)$ \\
\hline T5 (FSL) & $36.35(34.96-37.66)$ & $9.12(8.27-9.94)$ & $17.46(16.85-18.10)$ & $4.81(4.22-5.51)$ & $54.20(52.27-56.24)$ \\
\hline BART (ZSL) & $37.36(36.18-38.59)$ & $8.08(7.34-8.88)$ & $16.62(16.08-17.18)$ & $5.14(4.52-5.84)$ & $44.91(44.05-45.83)$ \\
\hline BART (FSL) & $\mathbf{4 0 . 8 6}(39.84-41.81)$ & $\mathbf{9 . 4 0}(8.69-10.08)$ & $18.38(17.93-18.85)$ & $6.06(5.46-6.68)$ & $53.96(53.17-54.69)$ \\
\hline PEGASUS (ZSL) & $26.36(25.05-27.64)$ & $5.01(4.38-5.70)$ & $14.69(13.95-15.34)$ & $2.18(1.83-2.58)$ & $65.52(60.81-70.36)$ \\
\hline PEGASUS (FSL) & $36.02(34.63-37.33)$ & $7.95(7.26-8.65)$ & $\mathbf{1 8 . 8 8}(18.27-19.49)$ & $5.21(4.57-5.85)$ & $74.29(71.73-76.92)$ \\
\hline
\end{tabular}

Table 1: Abstract multi-document summarization on DUC 2007 with $95 \%$ confidence intervals.

\subsection{Update Summarization at TAC 2009}

In 2009, the Text Analysis Conference (TAC) summarization evaluation explored summarizing sets of 10 newswire articles with respect to a given topic description in approximately 100 words under the assumption that a user had already read a given set of earlier articles (Dang and Owczarzak, 2009). Of 44 topics used in 2009, we used 30 for testing (with 10 for training and 4 for validation under FSL). Table 2 presents these results. While T5 had the highest performance in zero-shot performance, there was no statistically significant difference in terms of ROUGE after few-shot training, although T5 did obtain improved BLEU.

\begin{tabular}{lcrrrr}
\hline System & ROUGE-1 & ROUGE- & ROUGE-L & BLEU-4 \\
\hline T5 (ZSL) & $29.97(28.55-31.38)$ & $9.03(7.78-10.30)$ & $17.98(16.89-19.21)$ & $3.67(3.10-4.36)$ & $27.96(26.76-29.31)$ \\
T5 (FSL) & $38.36(36.92-39.85)$ & $\mathbf{1 1 . 5 6}(10.25-12.95)$ & $21.06(19.74-22.59)$ & $\mathbf{8 . 5 5}(7.32-9.78)$ & $33.91(32.75-35.03)$ \\
BART (ZSL) & $12.82(11.68-13.97)$ & $3.73(3.27-4.20)$ & $9.43(8.76-10.12)$ & $0.57(0.44-0.74)$ & $7.32(5.43-9.41)$ \\
BART (FSL) & $\mathbf{3 9 . 2 8}(38.10-40.46)$ & $11.33(10.37-12.44)$ & $21.11(20.27-22.02)$ & $7.30(6.49-8.11)$ & $45.30(44.24-46.33)$ \\
PEGASUS (ZSL) & $25.69(23.88-27.66)$ & $5.70(4.74-6.69)$ & $16.72(15.77-17.65)$ & $3.31(2.81-3.91)$ & $75.56(71.07-80.36)$ \\
PEGASUS (FSL) & $38.96(37.64-40.17)$ & $10.44(9.51-11.40)$ & $\mathbf{2 1 . 9 2 ( 2 0 . 8 4 - 2 2 . 9 5 )}$ & $7.00(6.24-7.88)$ & $43.59(41.50-45.87)$ \\
\hline
\end{tabular}

Table 2: Abstract multi-document summarization on TAC 2009 with $95 \%$ confidence intervals.

\subsection{Guided Summarization at TAC 2010}

Similar to the 2009 evaluation, the summarization track's goal in TAC 2010 was to produce 100 -word summaries of sets of 10 newswires articles for 46 given topics. However, in 2010 each topic was assigned to one of five pre-defined categories, and summaries were expected to cover all aspects associated with that category (e.g., for Accidents and Natural Disasters, summaries should cover (a) what happened, (b) when it happened, (c) the reasons for the accident or disaster, (d) casualties, (e) damages, and (f) rescue efforts or countermeasures) (Owczarzak and Dang, 2010). We used 30 topics for testing (with 10 for training and 6 for validation). Results are illustrated in Table 3. In this case, BART had the highest performance in both ZSL and FSL settings, although FSL provided significant improvements for all models, allowing T5 to obtain similar ROUGE-2 and ROUGE-L performance. 


\begin{tabular}{|c|c|c|c|c|c|}
\hline System & ROUGE-1 & ROUGE-2 & ROUGE-L & BLEU-4 & Repetition \\
\hline T5 (ZSL) & $27.01(25.65-28.35)$ & $6.25 \quad(5.35-7.29)$ & $15.72(14.84-16.75)$ & $2.06(1.72-2.45)$ & $30.47(29.07-31.91)$ \\
\hline T5 (FSL) & $34.13(32.72-35.77)$ & $8.36 \quad(7.32-9.50)$ & $17.35(16.44-18.28)$ & $5.59(4.70-6.54)$ & $32.60(31.25-34.05)$ \\
\hline BART (ZSL) & $28.97(27.48-30.70)$ & $6.32 \quad(5.58-7.24)$ & $15.64(14.80-16.40)$ & $3.62(3.11-4.22)$ & $27.96(26.06-29.74)$ \\
\hline BART (FSL) & $38.22(36.98-39.41)$ & $10.15(9.17-11.18)$ & $20.11(19.27-20.94)$ & $6.85(5.99-7.68)$ & $39.91(38.67-41.11)$ \\
\hline PEGASUS (ZSL) & $24.87(23.14-26.48)$ & $4.99(4.31-5.77)$ & $14.80(13.97-15.65)$ & $2.66(2.26-3.19)$ & $57.15(51.71-62.81)$ \\
\hline PEGASUS (FSL) & $36.31(34.95-37.63)$ & $9.21 \quad(8.27-10.15)$ & $20.35(19.56-21.26)$ & $5.81(5.09-6.62)$ & $40.39(37.73-43.31)$ \\
\hline
\end{tabular}

Table 3: Abstract multi-document summarization on DUC 2007 with $95 \%$ confidence intervals.

\subsection{MEDIQA Summarization}

The MEDIQA collection contains consumer health questions, sets of passages extracted from reliable websites relevant to the question, and human-authored multi-document summaries of the passages intended to provide consumer-friendly answers (Savery et al., 2020). Of the 156 available abstractive multi-document summaries, we used 141 questions for testing (with 10 for training and 5 for validation under FSL). Table 4 provides these results. While FSL provided a clear improvement for all models, there were no statistically significant differences in summary quality between the three models using FSL. Example summaries from all systems for a single MEDIQA question are provided in Figure 3.

\begin{tabular}{|c|c|c|c|c|c|}
\hline System & ROUGE-1 & ROUGE-2 & ROUGE-L & BLEU-4 & Repetition \\
\hline T5 (ZSL) & $31.09(28.46-33.72)$ & $14.63(11.77-17.58)$ & $22.52(20.15-25.19)$ & $7.12(5.07-9.36)$ & $31.00(29.06-32.96)$ \\
\hline T5 (FSL) & $\mathbf{3 8 . 5 6}(35.94-41.13)$ & $18.52(15.54-21.50)$ & $\mathbf{2 6 . 0 0}(23.67-28.76)$ & $10.90 \quad(9.08-13.07)$ & $36.19(34.73-37.78)$ \\
\hline BART (ZSL) & $33.51(31.21-36.14)$ & $13.87(11.52-16.31)$ & $20.87(18.92-22.88)$ & $8.21 \quad(6.38-10.18)$ & $38.24(36.60-39.79)$ \\
\hline BART (FSL) & $37.65(35.07-40.37)$ & $17.01(14.38-20.12)$ & $23.54(21.34-26.00)$ & $10.83(8.83-13.04)$ & $41.48(40.13-42.87)$ \\
\hline PEGASUS (ZSL) & $29.75(26.20-32.89)$ & $12.17(9.44-15.12)$ & $20.88(18.19-23.49)$ & $8.61(6.53-10.84)$ & $63.87(58.64-69.69)$ \\
\hline PEGASUS (FSL) & $37.02(33.86-40.33)$ & $17.04(13.95-20.12)$ & $24.90(22.18-27.68)$ & $12.40 \quad(9.96-15.08)$ & $46.81(43.40-50.27)$ \\
\hline
\end{tabular}

Table 4: Abstract multi-document summarization on MEDIQA with 95\% confidence intervals.

\subsection{Few-shot and Zero-shot Learning}

Figure 2 compares the performance of each model in FSL and ZSL settings. FSL provided significant increases in performance on all tasks for PEGASUS, all but MEDIQA for BART, and only two tasks for T5, suggesting that while FSL is clearly useful for all three models, it most benefits PEGASUS.
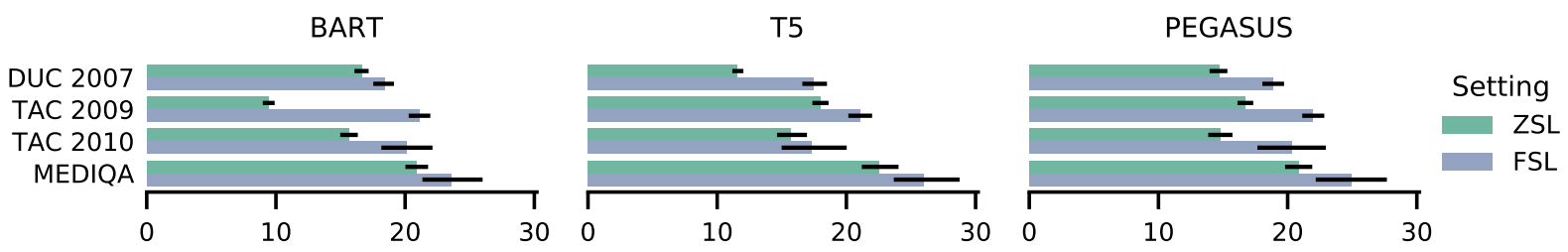

Figure 2: Rouge-L of each model trained under the few-shot (FSL) and zero-shot (ZSL) learning settings.

\section{Conclusion}

We evaluated the summarization quality produced by three state-of-the-art transformers: BART, T5, and PEGASUS on four challenging summarization dataset in both zero-shot and few-shot learning settings. Our results indicate that, while there are statistically significant differences between the models in zero-shot settings, after few-shot learning with as few as 10 examples, there is little discernible difference between them. This suggests that while large improvements have been made on standard single-document benchmarks, highly abstractive multi-document summarization remains challenging.

\section{Acknowledgements}

This work was supported by the intramural research program at the U.S. National Library of Medicine, National Institutes of Health, and utilized the computational resources of the NIH HPC Biowulf cluster (http://hpc.nih.gov). 
Question: What are the causes of childhood obesity?

Context: Overweight and Obesity (Risk Factors): There are many risk factors for overweight and obesity. Some risk factors can be changed, such as unhealthy lifestyle habits and environments. Other risk factors, such as age, family history and genetics, race and ethnicity, and sex, cannot be changed. Heathy lifestyle changes can decrease your risk for developing overweight and obesity. Unhealthy lifestyle habits Lack of physical activity, unhealthy eating patterns, not enough sleep, and high amounts of stress can increase your risk for overweight and obesity. Lack of physical activity Lack of physical activity due to high amounts of TV, computer, videogame or other screen usage has been associated with a high body mass index. [ABRIDGED]

Reference Summary: There are many factors that contribute to increasing your child's risk of becoming overweight. Regularly eating high calorie foods can cause your child to gain weight. Lack of physical exercise can contribute to childhood obesity. If your child comes from a family of overweight people, he or she may be more likely to put on weight. Personal, parental, and family stress can also increase a child's risk of obesity.

BART (FSL): There are many risk factors for overweight and obesity. Some risk factors can be changed, such as age, family history and genetics, race and ethnicity, and sex. However, some risk factors cannot be changed. These include: poor diet, lack of physical activity, unhealthy eating patterns, not enough sleep, and high amounts of stress. social factors such as having a low socioeconomic status or an unhealthy social or unsafe environment in the neighborhood. Eating too much saturated and trans fats, eating foods high in added sugars, and having an unhealthy eating pattern can increase your risk for obesity. Avoiding foods that are high in fat and saturated fat, eating smaller meals, and reducing screen time

BART (ZSL): question: what are the causes of childhood obesity summarize: Overweight and Obesity (Risk Factors): There are many risk factors for overweight and obesity. Some risk factors can be changed, such as unhealthy lifestyle habits and environments. Heathy lifestyle changes can decrease your risk for becoming overweight and obese, and can help you lose weight and maintain a healthy weight. Other risk factors, including age, family history and genetics, race and ethnicity, and sex, cannot be changed. Age Childhood obesity remains a serious problem in the United States, and some populations are more at risk for childhood obesity than others. The risk of unhealthy weight gain increases as you age. Adults who have

T5 (FSL): There are many risk factors for overweight and obesity. Some risk factors can be changed, such as unhealthy lifestyle habits and environments. Other risk factors such as age, family history and genetics, race and ethnicity, and sex, cannot be changed. Unhealthy lifestyle habits Lack of physical activity, unhealthy eating patterns, not enough sleep, and high amounts of stress can increase your risk for overweight or obesity. Lack of exercise due to TV, computer, videogame, or other screen usage has been associated with a high body mass index. Eating too much saturated and trans fats Eating foods high in added sugars Visit Heart-healthy eating for more information about healthy

T5 (ZSL): Unhealthy lifestyle habits Lack of physical activity, unhealthy eating patterns, not enough sleep, and high amounts of stress can increase your risk for overweight and obesity . age Childhood obesity remains a serious problem in the united states, and some populations are more at risk for childhood obesity than others

PEGASUS (FSL): Lifestyle changes can decrease your risk for developing overweight and obesity. Unhealthy lifestyle habits, such as eating more calories than you use, and not enough sleep can increase your risk for overweight and obesity. Lack of physical activity due to high amounts of TV, computer, videogame or other screen usage has been associated with a high body mass index. Healthy lifestyle changes, such as being physically active and reducing screen time, can help you aim for a healthy weight.

PEGASUS (ZSL): Unhealthy lifestyle habits Lack of physical activity, unhealthy eating patterns, not enough sleep, and high amounts of stress can increase your risk for overweight and obesity. Unhealthy environments Many environmental factors can increase your risk for overweight and obesity: social factors such as having a low socioeconomic status or an unhealthy social or unsafe environment in the neighborhood built environment factors such as easy access to unhealthy fast foods, limited access to recreational facilities or parks, and few safe or easy ways to get exercise. Read more Overweight and obesity can be prevented by eating healthy foods, getting enough sleep, and reducing stress. Read more Overweight and obesity can be prevented by eating healthy foods, getting enough sleep, and reducing stress. Read

Figure 3: Example summaries for the question, What are the causes of childhood obesity?

\section{References}

Hoa Trang Dang and Karolina Owczarzak. 2009. Overview of the TAC 2009 summarization track. In Proceedings of the Second Text Analysis Conference, volume 2, Gaithersburg, Maryland, USA. National Institute of Standards and Technology.

Jacob Devlin, Ming-Wei Chang, Kenton Lee, and Kristina Toutanova. 2019. BERT: Pre-training of deep bidirectional transformers for language understanding. In Proceedings of the 2019 Conference of the North American Chapter of the Association for Computational Linguistics: Human Language Technologies, Volume 1 (Long and Short Papers), pages 4171-4186, Minneapolis, Minnesota, June. Association for Computational Linguistics.

Li Dong, Nan Yang, Wenhui Wang, Furu Wei, Xiaodong Liu, Yu Wang, Jianfeng Gao, Ming Zhou, and HsiaoWuen Hon. 2019. Unified language model pre-training for natural language understanding and generation. In H. Wallach, H. Larochelle, A. Beygelzimer, F. d'Alché-Buc, E. Fox, and R. Garnett, editors, Advances in Neural Information Processing Systems 32, pages 13063-13075. Curran Associates, Inc. 
Xiangyu Duan, Mingming Yin, Min Zhang, Boxing Chen, and Weihua Luo. 2019. Zero-shot cross-lingual abstractive sentence summarization through teaching generation and attention. In Proceedings of the 57th Annual Meeting of the Association for Computational Linguistics, pages 3162-3172, Florence, Italy, July. Association for Computational Linguistics.

David Graff. 2002. The aquaint corpus of English news text: portions@ 1998-2000 New York Times, Inc.,(C) 1998-2000 Associated Press, Inc., (C) 1996-2000 Xinhua News Service. Linguistic Data Consortium.

Nitish Shirish Keskar, Bryan McCann, Lav R. Varshney, Caiming Xiong, and Richard Socher. 2019. CTRL: A conditional transformer language model for controllable generation.

Mike Lewis, Yinhan Liu, Naman Goyal, Marjan Ghazvininejad, Abdelrahman Mohamed, Omer Levy, Ves Stoyanov, and Luke Zettlemoyer. 2019. BART: Denoising sequence-to-sequence pre-training for natural language generation, translation, and comprehension.

Chin-Yew Lin. 2004. ROUGE: A package for automatic evaluation of summaries. In Text Summarization Branches Out, pages 74-81, Barcelona, Spain, July. Association for Computational Linguistics.

Peter J. Liu, Yu-An Chung, and Jie Ren. 2019a. SummAE: Zero-shot abstractive text summarization using length-agnostic auto-encoders.

Xiaodong Liu, Pengcheng He, Weizhu Chen, and Jianfeng Gao. 2019b. Multi-task deep neural networks for natural language understanding. In Proceedings of the 57th Annual Meeting of the Association for Computational Linguistics, pages 4487-4496, Florence, Italy, July. Association for Computational Linguistics.

Zhengyuan Liu, Ke Shi, and Nancy F. Chen. 2020. Conditional neural generation using sub-aspect functions for extractive news summarization.

Hans Peter Luhn. 1958. The automatic creation of literature abstracts. IBM Journal of Research and Development, 2(2):159-165.

Inderjeet Mani. 2009. Summarization evaluation: an overview. In Proceedings of the NTCIR Workshop, volume 2.

Karolina Owczarzak and Hoa Trang Dang. 2010. Overview of the TAC 2010 summarization track. In Proceedings of the Third Text Analysis Conference, volume 3, Gaithersburg, Maryland, USA. National Institute of Standards and Technology.

Kishore Papineni, Salim Roukos, Todd Ward, and Wei-Jing Zhu. 2002. Bleu: a method for automatic evaluation of machine translation. In Proceedings of the 40th Annual Meeting of the Association for Computational Linguistics, pages 311-318, Philadelphia, Pennsylvania, USA, July. Association for Computational Linguistics.

Colin Raffel, Noam Shazeer, Adam Roberts, Katherine Lee, Sharan Narang, Michael Matena, Yanqi Zhou, Wei Li, and Peter J. Liu. 2019. Exploring the limits of transfer learning with a unified text-to-text transformer.

Max Savery, Asma Ben Abacha, Soumya Gayen, and Demner-Fushman. 2020. Question-driven summarization of answers to consumer health questions. https://doi.org/10.17605/OSF.IO/FYG46.

TensorFlow Datasets, a collection of ready-to-use datasets. https://www . tensorflow. org/datasets.

Thomas Wolf, Lysandre Debut, Victor Sanh, Julien Chaumond, Clement Delangue, Anthony Moi, Pierric Cistac, Tim Rault, Rémi Louf, Morgan Funtowicz, and Jamie Brew. 2019. Huggingface's transformers: State-of-the-art natural language processing.

Zhilin Yang, Zihang Dai, Yiming Yang, Jaime Carbonell, Russ R Salakhutdinov, and Quoc V Le. 2019. XLNet: Generalized autoregressive pretraining for language understanding. In H. Wallach, H. Larochelle, A. Beygelzimer, F. d'Alché-Buc, E. Fox, and R. Garnett, editors, Advances in Neural Information Processing Systems 32, pages 5753-5763. Curran Associates, Inc.

Jingqing Zhang, Yao Zhao, Mohammad Saleh, and Peter J. Liu. 2019. Pegasus: Pre-training with extracted gap-sentences for abstractive summarization. 


\section{Appendix A Implementation Details}

Metrics, confidence intervals, and the PEGASUS implementation were provided by https://github. $\mathrm{com} /$ google-research/pegasus. All models were trained with a batch size of 8 , maximum sequence length of 512 tokens, and 3 warm-up epochs followed by 20 training epochs using single V100X GPUs (32 GB VRAM) on a shared cluster. Validation loss was measured every epoch and the snapshot with lowest validation loss was used for FSL evaluation. Documents were sorted by similarity to their reference summaries when provided to the model. T5 and BART implementations were provided by HuggingFace's Transformers package (Wolf et al., 2019). Existing datasets were obtained using the TensorFlow DataSets catalogue. The source code for this paper is available on GitHub at https://github. com/h4ste/mdas. 